\title{
Symmetry-projected wave functions in quantum Monte Carlo calculations
}

\author{
Hao Shi, ${ }^{1, *}$ Carlos A. Jiménez-Hoyos, ${ }^{2}$ R. Rodríguez-Guzmán, ${ }^{2,3}$ Gustavo E. Scuseria, ${ }^{2,3}$ and Shiwei Zhang ${ }^{1, \dagger}$ \\ ${ }^{1}$ Department of Physics, The College of William and Mary, Williamsburg, Virginia 23187, USA \\ ${ }^{2}$ Department of Chemistry, Rice University, Houston, Texas 77005, USA \\ ${ }^{3}$ Department of Physics and Astronomy, Rice University, Houston, Texas 77005, USA
}

(Received 31 January 2014; revised manuscript received 13 March 2014; published 31 March 2014)

\begin{abstract}
We consider symmetry-projected Hartree-Fock trial wave functions in constrained-path Monte Carlo (CPMC) calculations. Previous CPMC calculations have mostly employed Hartree-Fock (HF) trial wave functions, restricted or unrestricted. The symmetry-projected HF approach results in a hierarchy of wave functions with increasing quality: the more symmetries that are broken and restored in a self-consistent manner, the higher the quality of the trial wave function. This hierarchy is approximately maintained in CPMC calculations: the accuracy in the energy increases and the statistical variance decreases when further symmetries are broken and restored. Significant improvement is achieved in CPMC with the best symmetry-projected trial wave functions over those from simple HF. We analyze and quantify the behavior using the two-dimensional repulsive Hubbard model as an example. In the sign-problem-free region, where CPMC can be made exact but a constraint is deliberately imposed here, spin-projected wave functions remove the constraint bias. Away from half filling, spatial symmetry restoration in addition to that of the spin leads to highly accurate results from CPMC. Since the computational cost of symmetry-projected HF trial wave functions in CPMC can be made to scale algebraically with system size, this provides a potentially general approach for accurate calculations in many-fermion systems.
\end{abstract}

DOI: 10.1103/PhysRevB.89.125129

\section{INTRODUCTION}

Approximations able to account for the most relevant correlations in low-dimensional many-fermion systems represent a cornerstone in condensed-matter physics. In particular, very challenging phenomena such as the high- $T_{c}$ superconductivity [1], the colossal magnetic resistance [2], as well as the superconductivity in the iron-based compounds $[3,4]$ require us to better understand the nature of the electron-electron correlations and their impact on the resulting properties of the considered quantum systems. Within this context, the Hubbard model is regarded as a paradigm since, in spite of its essential simplicity, it displays several challenging properties associated with the relevant physics in strongly correlated many-electron systems. From the theoretical point of view, Hubbard-like models also represent valuable benchmark systems for testing different state-of-the-art approximations that can subsequently be extended to more realistic situations.

Nowadays there are several such approximations already used to study both the one- (1D) and two-dimensional (2D) Hubbard models with variable degrees of success. For sufficiently small lattices, exact diagonalization is possible. However, due to its exponential cost, such an exact diagonalization becomes impossible beyond a given system size. One can then resort to other approximations such as the variational Monte Carlo [5-7] (VMC), the coupled cluster [8] (CC), and the density matrix renormalization group [9-11] (DMRG) methods.

Auxiliary-field quantum Monte Carlo [12-14] (AFQMC) is one of the most popular methods to extract collective properties of quantum many-body systems. However, it is limited by a sign problem in Hubbard-like models, where the local interactions lead to auxiliary fields that are real, and

\footnotetext{
*boruoshihao@gmail.com

†shiwei@wm.edu
}

PACS number(s): 71.10.Fd, 02.70.Ss, 05.30.Fk, 71.27.+a

by a phase problem in realistic electronic systems, where the Coulomb interaction leads to complex fields [14]. In order to remove the exponential increase of the statistical noise with system size (or inverse temperature), we control the sign/phase problem approximately. The quantum Monte Carlo (QMC) process is formulated as open-ended random walks in the space of Slater determinants. A constraint, formally exact, is imposed on the paths of the random walk according to a sign or phase condition of the walker. This approach has been referred to as the constrained-path Monte Carlo [15,16] (CPMC) method in the case of "only" a sign problem and the phaseless AFQMC [17] for the general case with a phase problem. In practice, the constraint is implemented by the overlap of the sampled Slater determinants with a trial wave function. Despite its approximate nature, this approach is very accurate even with a simple mean-field trial wave function, as has been shown before in a wide range of applications [14,18-20].

In quantum mechanical systems, the symmetries of the Hamiltonian are crucial in characterizing properties such as the excitation spectrum, i.e., the basic fingerprint of the system [21-27]. We have studied [28] how the use of symmetry influences the CPMC method and AFQMC calculations in general, both in the form of the Hubbard-Stratonovich (HS) transformation and in the trial wave function. It was shown that imposition of symmetry properties resulted in a significant increase in the accuracy and efficiency in the QMC. Choosing a symmetry-adapted HS transformation often does not change the cost of the calculation significantly. On the other hand, symmetry adapting a trial wave function may result in expansions whose length depends on system size. In our prior work, we obtained symmetry-adapted wave functions by using a complete active space (CAS) expansion in the subspace of the open shell. The problem with CAS expansions is that the size of the wave function scales exponentially with the dimension of the active space.

An alternative to obtain highly correlated wave functions which, at the same time, respect the original symmetries 
of the considered many-fermion problem is provided by symmetry restoration via projection techniques. Very recently, a hierarchy of variational symmetry-projected approximations, inherited from nuclear structure physics [21], has been considered to describe the ground and excited states of the 1D (Ref. [29]) and 2D (Ref. [27]) Hubbard models. They have also been successfully incorporated into the description of molecular systems within the projected quasiparticle theory (PQT) framework [30,31]. A symmetry-projected HF wave function considers a Slater determinant $|\bar{\phi}\rangle$ which deliberately breaks several symmetries of the original Hamiltonian. By the superposition of the (degenerate) Goldstone manifold $\hat{R}|\bar{\phi}\rangle$, where $\hat{R}$ represents a symmetry operation, one can recover the desired symmetry in a fully self-consistent variation-afterprojection (VAP) procedure. In addition, the intrinsic Slater determinant $|\bar{\phi}\rangle$ resulting from the symmetry-projected VAP procedure contains defects that provide an intuitive physical picture regarding the basic units of quantum fluctuations [29]. Recently, symmetry-restored trial wave functions have been used in CPMC in the study of nuclear shell models [32].

It is known that symmetry projection out of a Slater determinant results in a wave function that is not size extensive [31], i.e., the correlation energy recovered by such an ansatz does not scale linearly with system size. This is also true for the CAS expansions we have considered before. Symmetry-projected wave functions can, nevertheless, account for some of the strong correlations (due to the degeneracies in the single-particle spectrum). In this paper, we examine the use of symmetry-projected wave functions as trial states within the CPMC framework. We stress that even though a single symmetry-broken Slater determinant is used in the trial wave function, the symmetry-projected wave functions are, via the projection operators, multideterminant in nature. In addition, the symmetry-projected state can access highly excited configurations. In this way, they bring a very sophisticated trial ansatz which can be expected to improve the quality of the sign/phase constraint in CPMC. Indeed, our results show that the use of such trial wave functions greatly improves the CPMC results. The combined approach demonstrates dramatically better behavior that successfully removes the size-extensivity problem of projected HF. Often the full symmetry-projected trial wave function eliminates the systematic error from the constraint, and the CPMC calculations approach the exact answer, with a reduced variance.

\section{METHOD}

In this work, we use as a benchmark system the twodimensional repulsive Hubbard model, which is written in second-quantized form as [33]

$$
\hat{H}=\hat{K}+\hat{V}=-t \sum_{\langle\mathbf{i}, \mathbf{j}\rangle \sigma}^{L} c_{\mathbf{i} \sigma}^{\dagger} c_{\mathbf{j} \sigma}+U \sum_{\mathbf{i}}^{L} n_{\mathbf{i} \uparrow} n_{\mathbf{i} \downarrow},
$$

where the first term represents kinetic energy from electron hopping $(t>0)$ and the second is the repulsive on-site interaction $(U>0)$. The operators $c_{\mathbf{i} \sigma}^{\dagger}$ and $c_{\mathbf{i} \sigma}$ create and annihilate an electron with spin direction $\sigma$ on the ith lattice site. Note that we have introduced a vector notation for the site index, i.e., $\mathbf{i}=\left(i_{x}, i_{y}\right)$. The operators $\hat{n}_{\mathbf{i} \sigma}=\hat{c}_{\mathbf{i} \sigma}^{\dagger} \hat{c}_{\mathbf{i} \sigma}$ are the local number operators. The notation $\langle\mathbf{i}, \mathbf{j}\rangle$ is used to denote that only hopping between nearest-neighbor sites is allowed. We assume periodic boundary conditions (PBC) in both the $x$ and $y$ directions. Throughout this paper, energies will be reported in units of $t$ and we set $t=1$.

\section{A. Symmetry-projected wave function}

In this section, we briefly discuss the form of the symmetryprojected HF states used as trial wave functions. For more details of the symmetry-projected approach, we refer the reader to our previous works (Refs. [29] and [27]).

We work with HF-type Slater determinants which preserve the number of electrons in the system. An arbitrary HF-type Slater determinant can, in general, break several symmetries of the original Hamiltonian. Typical examples are the rotational (in spin space) and spatial (space group for periodic systems) symmetries. In the present study, we have considered two different kinds of symmetry-broken states: unrestricted (UHF) configurations which are eigenstates of $S_{z}$ and therefore have a definite $N_{\uparrow}$ and $N_{\downarrow}$, as well as generalized (GHF) configurations that break $S_{z}$ and therefore can only be characterized by an overall $N$ number of electrons.

To restore the spin quantum numbers, we use the spinprojection operator

$$
\hat{P}_{\Sigma \Sigma^{\prime}}^{S}=\frac{2 S+1}{8 \pi^{2}} \int d \Omega \mathcal{D}_{\Sigma \Sigma^{\prime}}^{S *}(\Omega) \hat{R}(\Omega),
$$

where $\hat{R}(\Omega)=e^{-i \alpha \hat{S}_{z}} e^{-i \beta \hat{S}_{y}} e^{-i \gamma \hat{S}_{z}}$ is the rotation operator in spin space, the label $\Omega=(\alpha, \beta, \gamma)$ stands for the set of Euler angles, and $\mathcal{D}_{\Sigma \Sigma^{\prime}}^{S}(\Omega)$ are Wigner functions. We note that if UHF wave functions are used, then the numerical effort can be alleviated significantly in the evaluation of matrix elements, as both the integrals over $\alpha$ and $\gamma$ can be carried out analytically.

To recover the spatial symmetries, we use the generic projection operator

$$
\hat{P}_{m m^{\prime}}^{k}=\frac{l}{h} \sum_{g} \Gamma_{m m^{\prime}}^{k *}(g) \hat{R}(g),
$$

where $\Gamma^{k}$ is an irreducible representation (irrep), which can be found by standard methods, and $\hat{R}(g)$ represents the corresponding symmetry operations in the considered lattices parametrized in terms of the label $g$. In addition, $l$ is the dimension of the irrep and $h$ is the order of the group in Eq. (3). We note that for the periodic Hubbard 2D system, the irreducible representations associated with the spatial symmetry can be characterized by the linear momentum $(k)$ inside the Brillouin zone and, for certain high-symmetry momenta, by additional parities under $x, y$, and $x-y$ reflections. In what follows, we will explicitly provide the linear momentum of the recovered irrep and, where appropriate, we will further provide the parities of the recovered state (if the full space-group projection is carried out).

We then superpose the Goldstone manifolds due to spin and/or spatial symmetries via the following ansatz:

$$
\left|\Psi_{K}^{\Theta}\right\rangle=\sum_{K^{\prime}} f_{K^{\prime}}^{\Theta} \hat{P}_{K K^{\prime}}^{\Theta}|\bar{\phi}\rangle,
$$

where $f_{K^{\prime}}^{\Theta}$ are variational parameters and $|\bar{\phi}\rangle$ is the reference HF-type single Slater determinant. Here, the indices $\Theta$ and $K$ 
denote quantum numbers associated with spin and/or spatial symmetries. Let us stress that through the action of the projection operator $\hat{P}_{K K^{\prime}}^{\Theta}$, the multideterminantal character of the state characterized by the quantum numbers $\Theta$ and $K^{\prime}$ is recovered and written in terms of the quantum numbers $\Theta$ and $K$. The linear combination introduced in Eq. (4) guarantees the independence of $\left|\Psi_{K}^{\Theta}\right\rangle$ with respect to rotations of the Slater determinant $|\bar{\phi}\rangle$.

The wave functions $\left|\Psi_{K}^{\Theta}\right\rangle$ of Eq. (4) are precisely the ones used in the present study as trial states within the CPMC scheme. The wave functions, given by Eq. (4), are determined by resorting to the Ritz variation of the projected energy with respect to both the mixing coefficients $\left(f_{K^{\prime}}^{\Theta}\right)$ and the HF-type determinant $|\bar{\phi}\rangle$. We stress that the wave functions are fully optimized in the presence of the projection operations, i.e., a VAP approach is used. In our previous work [29], we have discussed how the optimized determinant $|\bar{\phi}\rangle$ develops defects that can be interpreted as the basic units of quantum fluctuations in the system. We refer the reader to Ref. [27] for a discussion of how the optimization procedure is carried out in practice.

Before concluding this section, let us introduce the notation used in this paper for the symmetry-projected wave functions of Eq. (4):

(i) LM (linear momentum) is used to denote wave functions in which the linear momentum has been broken and restored.

(ii) SG (space group) is used for wave functions in which the full space group of the 2D square lattice has been broken and restored.

(iii) $S_{z}$ is used to indicate that $S_{z}$ projection has been done (out of a GHF-type determinant).

(iv) $\mathrm{S}$ (spin) is used to indicate that full spin projection has been carried out. If the determinant is of the GHF type (noncollinear), it will involve the triaxial integration of Eq. (2).

For example, SG,S-UHF means that the trial wave function was prepared by breaking and restoring the spin and space group from a UHF-type determinant, while LM, $S_{z}-\mathrm{GHF}$ indicates that linear momentum and $S_{z}$ have been broken and restored out of a GHF-type determinant. In both cases, the determinant is variationally optimized to minimize the energy in the presence of the symmetry-projection operators, and before introducing the Monte Carlo procedure described in the next section. We note that if the Hamiltonian breaks spin and has no spatial symmetry, there is often still timereversal and/or particle-number symmetry. In the case where the Hamiltonian breaks all symmetries, the standard HF or Hartree-Fock-Bogoliubov (HFB) wave function would result.

\section{B. СРMC}

We briefly summarize the ground-state CPMC method $[15,16]$ below to facilitate the ensuing discussions. The reader is referred to Ref. [14] and references therein for further details. Aside from the symmetry-projected trial wave function, there is an algorithmic advance in the present study, namely we have generalized the AFQMC approach to have GHF-type random walkers, as discussed in Sec. IIIB. This will allow the application of CPMC and AFQMC, in general, to Hamiltonians that do not conserve $\hat{S}_{z}$, for example one that includes spin-orbit coupling.
All ground-state AFQMC methods are based on the projection

$$
\left|\Psi_{0}\right\rangle \propto \lim _{\beta \rightarrow \infty} e^{-\beta\left(\hat{H}-E_{T}\right)}\left|\Psi_{T}\right\rangle,
$$

where $E_{T}$ is a trial ground-state energy (an initial guess which is then improved in the calculation) and $\left|\Psi_{T}\right\rangle$ is a guess of the ground-state wave function, i.e., a trial wave function, which is typically taken to be a single Slater determinant or a linear combination of Slater determinants. $\left\langle\Psi_{0} \mid \Psi_{T}\right\rangle \neq 0$ needs to be satisfied in order to project to the ground state. In a numerical method, the limit can be obtained iteratively by

$$
\left|\Psi^{(n+1)}\right\rangle=e^{-\Delta \tau \hat{H}}\left|\Psi^{(n)}\right\rangle
$$

where $\left|\Psi^{(0)}\right\rangle=\left|\Psi_{T}\right\rangle$ and $\Delta \tau$ is a small positive number.

The Trotter-Suzuki breakup [34,35] and a so-called Hubbard-Stratonovich (HS) transformation are used to break the propagator into many one-body propagators [14],

$$
e^{-\Delta \tau \hat{H}}=\int d \mathbf{x} p(\mathbf{x}) \hat{B}(\mathbf{x}),
$$

where $p(\mathbf{x})$ is a probability density function, for example, a uniform distribution of one Ising field per site $\left(x_{\mathbf{i}}= \pm 1\right.$ with $\mathbf{i}=1,2, \ldots, L$.). The one-body propagator $\hat{B}(\mathbf{x})$ in Eq. (7) has a special form, namely, a product of the exponential of one-body operators,

$$
\hat{B}(\mathbf{x})=e^{-\Delta \tau \hat{K} / 2} e^{\hat{v}(\mathbf{x})} e^{-\Delta \tau \hat{K} / 2},
$$

where $\hat{v}(\mathbf{x})$ is a one-body operator whose matrix elements are simple functions of the HS fields and of magnitude $O(\sqrt{\Delta \tau})$. Note that $\hat{K}$ is diagonal in momentum space, while $\hat{v}(\mathbf{x})$ is diagonal in real space with the Hirsch spin HS [36], which will be used throughout this paper.

By applying each one-body propagator to a Slater determinant wave function, we generate another Slater determinant [37]. The many-dimensional integral/sum over the auxiliary Ising fields (for each component of $\mathbf{x}$ and at each imaginary-time iteration $n$ ) is performed by Monte Carlo sampling the fields. The resulting linear combination of Slater determinants at each iteration $n$ gives a stochastic representation of the wave function $\left|\Psi^{(n)}\right\rangle$. After convergence, all of the sampled determinants from $n \geqslant n_{\text {eq }}$ can be used collectively to represent the ground-state wave function. The determinants have to be stabilized periodically with, for example, a modified Gram-Schmidt orthogonalization procedure [38].

CPMC uses importance sampling to steer the sampling toward more "likely" auxiliary-field configurations. This is achieved by a similarity transformation with a trial wave function $\left\langle\psi_{T} \mid \phi\right\rangle$ to modify the probability density function $p(\mathbf{x})$ in Eq. (7). The importance sampling does not affect the average values of the computed observables, only the variance. The better the trial wave function $\left|\psi_{T}\right\rangle$, the smaller the statistical error for a fixed amount of Monte Carlo samples. Since the CPMC process is a branching random walk, population control has to be applied periodically [15,39]. As a manifestation of the variance reduction, the extent of weight fluctuation (branching) will be reduced with a better $\left|\psi_{T}\right\rangle$. The use of symmetry-projected trial wave functions leads to a reduction of the statistical variance, as will be illustrated below. 
The sign problem [14] is the leading difficulty in QMC simulations of many-fermion systems. The problem occurs because the projection is, in general, symmetric about $|\phi\rangle$ and $-|\phi\rangle$. In the random walks, there is no mechanism to distinguish random walkers of opposite overall signs. In the course of the projection, if we switched the sign of each random walker (e.g., by exchanging two orbitals in $\left|\phi_{\uparrow}\right\rangle$, or $\left|\phi_{\downarrow}\right\rangle$, in a UHF-type simulation, or two spin orbitals in a GHF-type simulation), there would be no noticeable change and the sampled population would give an overlap of opposite sign with any trial wave function. In the half-filling case of the Hamiltonian in Eq. (1), it turns out that symmetry in the propagator and in $\left|\Psi_{T}\right\rangle$ can make the sign of the overlap remain non-negative, which eliminates the sign problem. In general, however, the overlap with any trial wave function (or with $\left|\Psi_{0}\right\rangle$ ) will be zero when averaged over imaginary time (or over the entire population at any large $n$ ), leading to infinite Monte Carlo variance. The sign problem can be controlled, exactly, by eliminating the walkers whose overlap with the ground state becomes zero [14],

$$
\left\langle\Psi_{0} \mid \phi\right\rangle=0,
$$

since they will contribute zero at any future projections. In practice, in place of the exact ground-state wave function, a trial wave function $\left|\psi_{T}\right\rangle$ is used instead to implement the constraint above,

$$
\left\langle\Psi_{T} \mid \phi\right\rangle=0,
$$

which will introduce a systematic bias in the results. The importance sampling transformation imposes this condition naturally, terminating random-walk paths that lead to a zero overlap with $\left|\Psi_{T}\right\rangle$. As mentioned, the systematic error of the constraint tends to be very small even with simple mean-field trial wave functions. We will show below that with the symmetry-projected wave function, the constrained-path bias is further reduced and becomes negligible in most of the systems studied.

\section{RESULTS}

\section{A. Half filling}

We first discuss the use of symmetry-projected trial wave functions for the half-filled Hubbard model. Since there is no sign problem in this case, CPMC calculations can be made exact by redefining the important sampling to have a nonzero minimum $[15,16]$. If we ignore this and apply the usual importance sampling with $\left|\Psi_{T}\right\rangle$ literally, the random walks can be constrained to a part of Slater determinant space because the paths are terminated by the condition in Eq. (10). The CPMC results will display a constraint bias. In this section, we will use the half-filled case in the artificial way with the constraint as a benchmark system to study the effect of the symmetry-projected trial wave function. Because the exact result is accessible, this study allows us to systematically examine the effect of the different symmetries in $\left|\Psi_{T}\right\rangle$.

Figure 1 is an illustration in the $4 \times 4$ Hubbard model at half filling $\left(N_{\uparrow}=N_{\downarrow}=8\right)$ with $U=4$. Here, the ground state corresponds to a spin singlet with momentum $(0,0)$ and even parity under all reflections. As we see from Fig. 1(b), CPMC with a UHF trial wave function has a bias in the
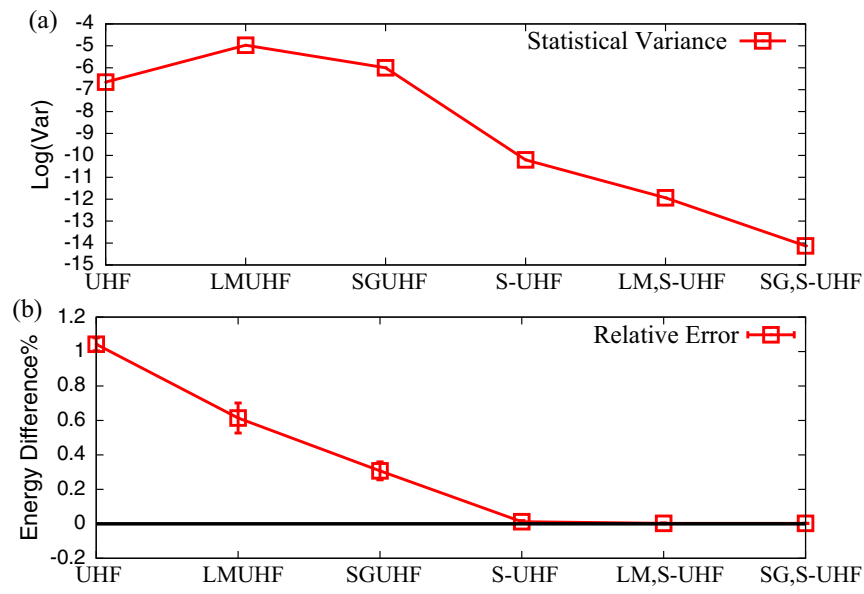

FIG. 1. (Color online) Performance of different symmetryprojected trial wave functions in CPMC. (a) The statistical uncertainty of the computed ground-state energy in a semilog plot (arbitrary scale). (b) The percentage error, $\left(E_{\mathrm{CP}}-E_{0}\right) /\left|E_{0}\right|$, between the CPMC energy and the exact energy. The system is the $4 \times 4$ Hubbard model, with $N_{\uparrow}=N_{\downarrow}=8$ and $U=4$. The number of walkers was 1000, an equilibrium phase of $\beta_{\mathrm{eq}}=4$ was discarded, and $\Delta \tau=0.01$ was used in the runs.

ground-state energy, with the calculated energy higher than the exact result $(-13.62185)$ by about $\sim 1 \%$. As more symmetries are included in the projected UHF-based wave function, the bias is seen to decrease, as well as the corresponding Monte Carlo variance, shown in Fig. 1(a). The only exception is in the variance from the UHF trial wave function, which falls slightly smaller than those from LMUHF and SGUHF trial wave functions. The reduction of energy bias and energy variance indicates that the quality of the trial wave function is improving. This is born out by their variational energies, which decrease monotonically following the sequence from left to right (not shown).

We also see from Fig. 1 that the CPMC energy becomes indistinguishable with the exact one as long as the spin symmetry is imposed. This is consistent with the findings from our previous studies of symmetry-preserving trial wave functions which were obtained from a CAS approach by diagonalizing a truncated active space [28].

We next study the behavior of the simulation as a function of system size. The results are summarized in Fig. 2, which shows the calculated energy per site from various approaches versus the inverse linear dimension of the square lattice. Two types of trial wave functions are compared: the standard UHF and the spin-symmetry (onto a singlet) projected S-UHF. Other systematic errors, such as Trotter error and population control bias, have been reduced by either systematic extrapolation or by very conservative choices of run parameters, and can be ignored. As mentioned earlier, symmetry-projected HF wave functions are not size extensive. The variational energy (per site) of S-UHF is considerably lower than that of UHF for smaller lattice sizes, but gradually approaches the UHF value as the system size is increased. As the fits indicate, the two give the same result in the thermodynamic limit, $E_{\mathrm{UHF}} / L \sim$ -0.797 . 


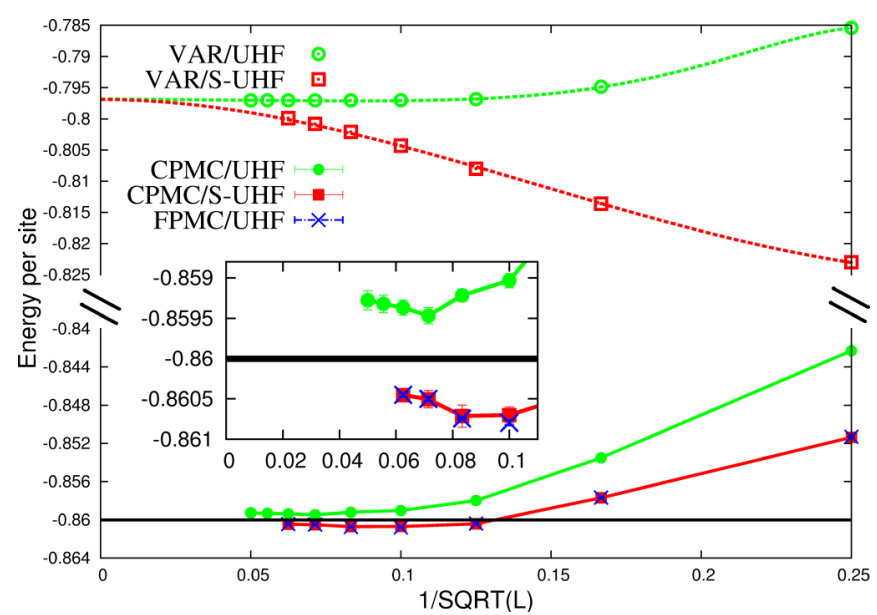

FIG. 2. (Color online) Size dependence of the computed energies in the half-filled Hubbard model at $U=4$. The behaviors of two different forms of trial wave functions are compared. The variational energies of the trial wave functions are shown in the upper curves. The dashed lines are polynomial fits to the data. CPMC results using the two trial wave functions as (artificial) constraints are shown in the lower curves, together with exact free-projection results. (Note the different vertical scales between the upper and lower curves.) Our best estimate of the ground-state energy in the thermodynamic limit is given by the horizontal lines. Its statistical uncertainty is indicated by the width of the line in the inset.

The CPMC results with an artificial constraint using UHF are shown as CPMC/UHF. Exact results from free projection (i.e., by "lifting" the constraint) [28] shown as FPMC/UHF are given for systems up to $16 \times 16$. The $\mathrm{CPMC/UHF}$ bias is visible at all lattice sizes. The largest discrepancy is seen between the two at the smallest lattice size (rightmost point), corresponding to a $1 \%$ error in Fig. 1. Convergence of the ground-state energy with system size is not monotonic under periodic boundary conditions, as seen in the bottom set of curves and the inset. To accurately determine the exact ground-state energy in the thermodynamic limit, we have done calculations on up to $14 \times 14$ lattices with twist-average boundary conditions, which gives much smaller finite-size effects than PBC. A polynomial fit [40] with a leading term of $1 / L^{3 / 2}$ was performed on the data (not shown), and our best estimate of the energy per site for the half-filled Hubbard model at $U=4$ as $L \rightarrow \infty$ is $E_{0} / L=-0.8600(1)$. The CPMC/UHF results are seen to converge to a value slightly higher than the exact energy in the thermodynamic limit.

CPMC using the symmetry-projected S-UHF trial wave function is seen to agree with exact free projection at all finite lattice sizes studied. This extends the conclusion from Fig. 1 that trial wave functions with spin symmetry have negligible bias to much larger supercells. These data indicate that despite the lack of size extensivity in the S-UHF trial wave function, CPMC seems to restore a consistent behavior as a function of system size.

\section{B. Away from half filling}

We next move away from half filling to examine the effect of the symmetry-projected HF trial wave function on the sign
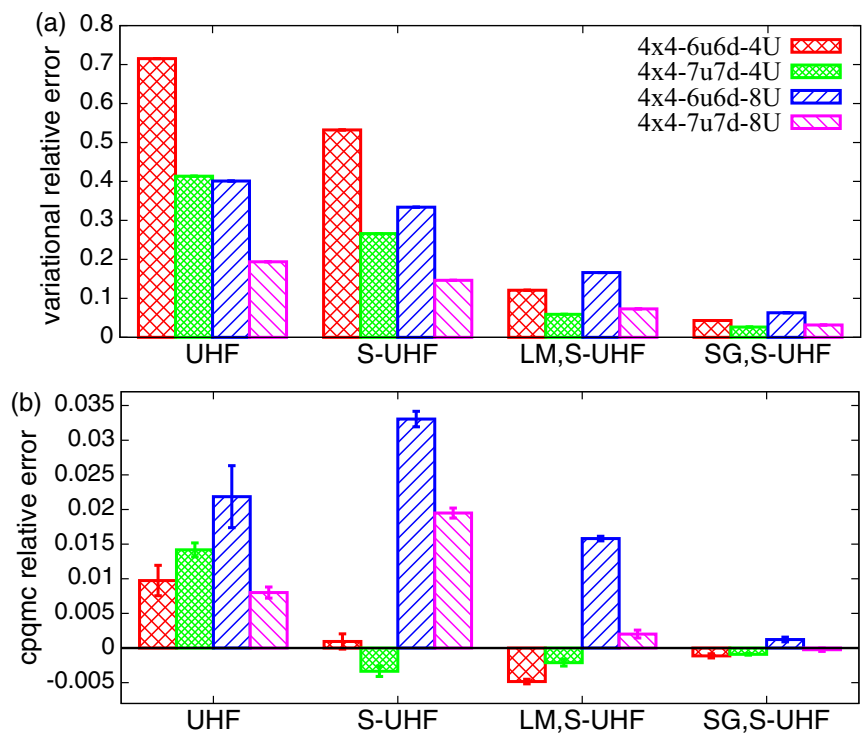

FIG. 3. (Color online) (a) Variational energies of the UHF and three UHF-based symmetry-projected wave functions, and (b) the corresponding CPMC energies using these as trial wave functions. The energies are shown in terms of relative errors in the correlation energy (see text). Note the different scales between (a) and (b). Four systems are studied, each represented by a different color/pattern of vertical bar. The CPMC results were obtained with a population size of 1000 and 10 independent blocks of size $\beta \sim 1$ each. The statistical error bars are indicated. Trotter error is negligible.

problem. We study the system close to half filling, which is the region that has the most severe sign problem [41]. Intermediate values of the interaction strength $U$ are chosen, comparable to the bandwidth, where the model is potentially most relevant to strongly correlated materials such as high- $T_{c}$ superconductors.

Figure 3 shows the results in $4 \times 4$ lattices where exact diagonalizations are available for comparison. Two filling values, $N_{\uparrow}=N_{\downarrow}=6$ and 7 , are each studied at $U=4$ and 8 . The ground state in all cases is a spin singlet with momentum $(0,0)$ transforming as the $B_{1}$ irrep of the $C_{4 v}$ group, save for the 12-electron system at $U=8$, where the ground state switches to the $A_{1}$ irrep. The variational energies for the UHF wave functions and three symmetry-projected HF wave functions are shown in Fig. 3(a), while the corresponding CPMC energies are shown in Fig. 3(b). Here the energies are shown in terms of relative errors in the correlation energy, defined as $\left(E-E_{0}\right) /\left(E_{\mathrm{RHF}}-E_{0}\right)$, where $E$ is the calculated energy (either variational or CPMC), $E_{0}$ is the exact result, and $E_{\mathrm{RHF}}$ is the restricted HF (equivalent to the Fermi-gas wave function) energy. The variational energy improves as more symmetries are broken and restored in the trial wave function, as expected. The corresponding CPMC using UHF trial wave functions yields relative errors in the computed correlation energy of a few percent, while CPMC with symmetry-projected trial wave functions, in general, has significantly smaller errors. CPMC/SG,S-UHF is the most accurate for the four systems studied here, with relative errors of about $0.1 \%$ in the correlation energy. In terms of percentage errors of the total energy (as plotted in Fig. 1), the $U=4$ systems have errors of $\sim-0.02 \%$ and the maximum error is 


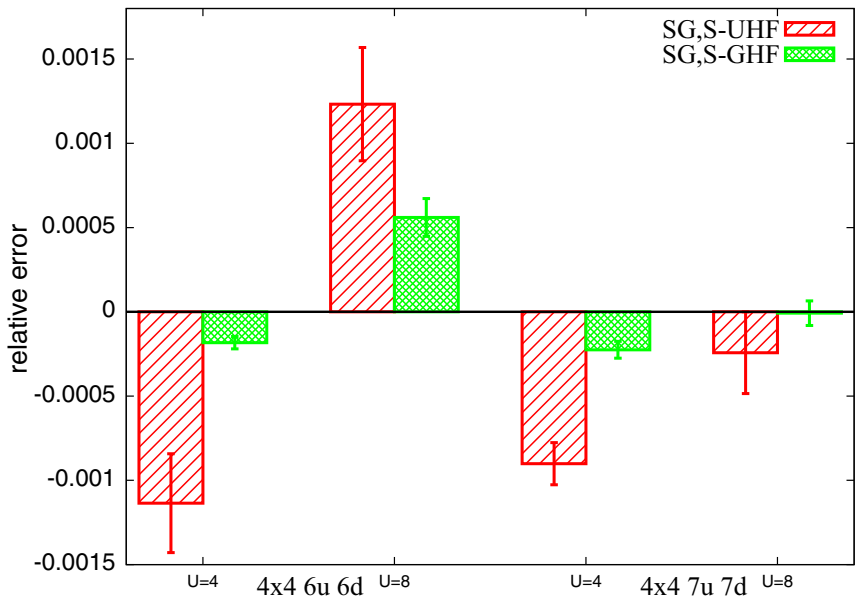

FIG. 4. (Color online) Illustration of GHF-based symmetryprojected trial wave functions in CPMC. The relative errors in the correlation energy from CPMC using GHF-type wave functions (SG,S-GHF) are compared with the corresponding UHF-type (SG,SUHF). The latter are taken from the rightmost group in Fig. 3. The same computational parameters are used for the two types of calculations.

$0.07 \%$ at $U=8$. Note that the ground-state energy in CPMC is calculated with the so-called mixed estimate, which is not variational [42].

We have also investigated the effect of GHF-based symmetry-projected wave functions. In the usual CPMC calculations discussed thus far, the random walkers have a form $\left|\phi_{\uparrow}\right\rangle \otimes\left|\phi_{\downarrow}\right\rangle$, where the $\uparrow$ - and $\downarrow$-spin determinants have $N_{\uparrow}$ and $N_{\downarrow}$ orbitals, each specified by $L$ amplitudes, as in UHF. The one-body propagators in Eq. (8) decouple into $\uparrow$ - and $\downarrow$-spin components which operate on $\left|\phi_{\uparrow}\right\rangle$ and $\left|\phi_{\downarrow}\right\rangle$, respectively. In the GHF type, a walker $|\phi\rangle$ contains $\left(N_{\uparrow}+N_{\downarrow}\right)$ spin orbitals, each of which is specified by $2 L$ amplitudes that evolve stochastically. The one-body propagators are given by $2 N \times 2 N$ matrices. In the present study, the form of the initial/trial wave function dictates which of the two approaches is used.

Since there is more freedom in wave functions of the symmetry-projected GHF form [29], a lower variational energy can be obtained. Note that lower energies may only be obtained when the symmetry-projected states are optimized in the presence of the projection operators (a VAP approach). This is seen from Fig. 4, which compares CPMC results from GHF-based and UHF-based trial wave functions with the same symmetry projections. A significant further reduction is seen in the CPMC results with GHF-based trial wave functions, leading to essentially exact energies. In Table I, we summarize all the CPMC ground-state energies with both types of trial wave functions.

The improvements in the energy from CPMC using symmetry-projected HF wave functions as the constraint show that these wave functions give better descriptions of the ground-state properties than standard mean field. One can then expect that such wave functions will also improve the calculations of other observables. We study this in Fig. 5 with the example of momentum distribution. In CPMC calculations of the expectation of observables which do not commute with the Hamiltonian, the back-propagation technique $[15,43]$ is almost always used to obtain a "pure" estimator, $\left\langle\Psi_{T}\left|e^{-\beta_{\mathrm{BP}} H} \hat{O}\right| \Psi_{0}\right\rangle /\left\langle\Psi_{T}\left|e^{-\beta_{\mathrm{BP}} H}\right| \Psi_{0}\right\rangle$, as opposed to the mixed estimate (corresponding to $\beta_{\mathrm{BP}}=0$ ) which is exact for the energy but biased for a general operator $\hat{O}$. For reasonable choices of the back-propagation time $\beta_{\mathrm{BP}}$, the technique gives exact estimators of any $\langle\hat{O}\rangle$ except for constrained-path errors. Here, for illustrative purposes, we use the mixed estimate to calculate $n(\mathbf{k})$, which helps to magnify the effect of $\left\langle\Psi_{T}\right|$.

As seen from Fig. 5, the calculated momentum distribution is incorrect around the Fermi energy when the UHF trial wave function is used. This is consistent with the fact that the UHF is a very poor wave function, and back propagation must be employed for observables when such trial wave functions are used. We see that the S-UHF trial wave function leads to little improvement, despite having improved the CPMC energy. As more symmetries are included in the projected $\mathrm{HF}$ wave functions, the results improve. The largest step occurs when the space group is added. This seems reasonable given the open-shell nature of this system. The result with the full SG,S-GHF trial wave function is essentially indistinguishable from exact diagonalization.

In Table II, several CPMC results are shown using symmetry-projected HF trial wave functions for larger systems away from half filling. Accurate energies from constraint release calculations [28] with the complete active-space selfconsistent field (CASSCF) wave function are available in these systems, which we use for a benchmark and are shown in the last column. The UHF trial wave functions are very good at these fillings and are often difficult to surpass [28], so it is significant that the symmetry-projected trial wave functions except the minimal set (spin symmetry only) perform better. In fact, the best results are consistent with the constraint-release results, while improving the statistical error by almost an order of magnitude.

Computational scaling with system size is an important consideration for any many-fermion method. In a CPMC

TABLE I. CPMC ground-state energies with UHF and various symmetry-projected trial wave functions. Two fillings (first column) and $U$ values are studied on a $4 \times 4$ lattice Hubbard model. The exact results in the last column are from exact diagonalization. The statistical error bars in the CPMC results are on the last digit and shown in parentheses.

\begin{tabular}{lllllllll}
\hline \hline System & $U$ & \multicolumn{1}{c}{ UHF } & S-UHF & LM,S-UHF & SG,S-UHF & SG, $S_{z}$-GHF & SG,S-GHF & Exact \\
\hline $6 \uparrow 6 \downarrow$ & 4 & $-17.703(6)$ & $-17.727(3)$ & $-17.7428(9)$ & $-17.7327(8)$ & $-17.7316(4)$ & $-17.7301(1)$ & -17.7296 \\
$6 \uparrow 6 \downarrow$ & 8 & $-14.73(4)$ & $-14.63(1)$ & $-14.784(3)$ & $-14.914(3)$ & $-14.907(2)$ & $-14.920(1)$ & -14.925 \\
$7 \uparrow 7 \downarrow$ & 4 & $-15.688(4)$ & $-15.758(3)$ & $-15.753(2)$ & $-15.7482(5)$ & $-15.7500(8)$ & $-15.7455(2)$ & -15.7446 \\
$7 \uparrow 7 \downarrow$ & 8 & $-11.77(1)$ & $-11.628(9)$ & $-11.844(7)$ & $-11.872(3)$ & $-11.847(3)$ & $-11.8689(9)$ & -11.8688 \\
\hline \hline
\end{tabular}




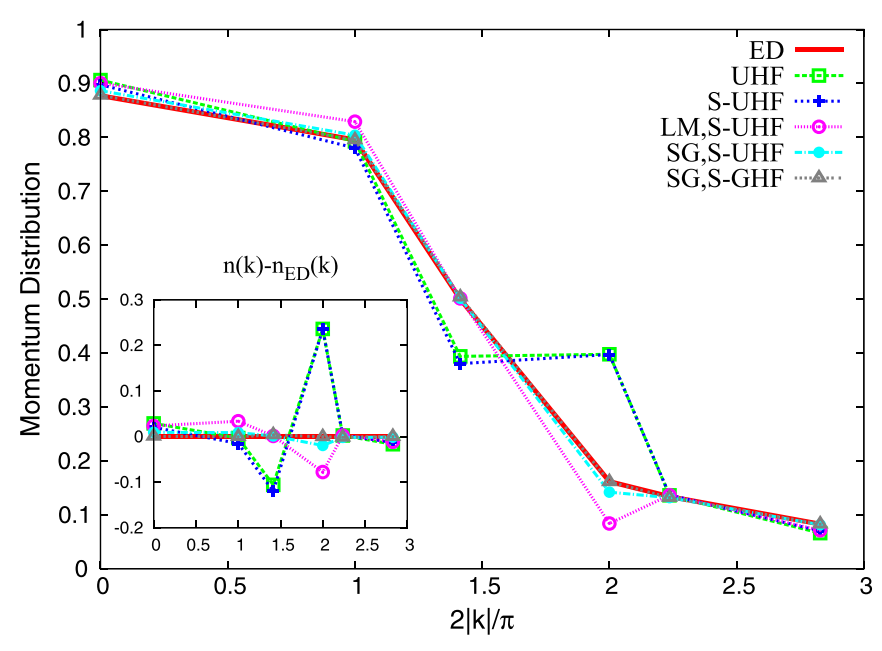

FIG. 5. (Color online) Momentum distribution $n(\mathbf{k})$ vs $|\mathbf{k}|$. Results from CPMC using different trial wave functions are compared with each other and with exact diagonalization (ED). The inset shows the error from ED results. The mixed estimator is used instead of back propagation to magnify the effect of the trial wave function. The system is $4 \times 4$ with $7 \uparrow, 7 \downarrow$ and $U=8$. The same run parameters are used as in Fig. 3.

calculation, the primary objects involving the trial wave function that need to be calculated repeatedly are the overlap $\left\langle\Psi_{T} \mid \phi\right\rangle$ and the one-body Green function $\left\langle\Psi_{T}\left|c_{\mathbf{k}}^{\dagger} c_{\mathbf{l}}\right| \phi\right\rangle /\left\langle\Psi_{T} \mid \phi\right\rangle$. For a single determinant $\left|\Psi_{T}\right\rangle$ such as UHF, the computations [14] scale like regular mean-field calculations. For a $\left|\Psi_{T}\right\rangle$ in the form of Eq. (4), a straightforward way is to expand it as a linear combination of Slater determinants after the reference determinant and variational parameters have been determined. This is the approach we have taken in the present paper as a proof-of-concept study of the effectiveness of such wave functions. However, the number of Slater determinants in such an expansion grows rapidly with system size. For example, in the $8 \times 8$ calculation in Table II, the SG,S-UHF trial wave function involved 14336 determinants. An alternative is to use Eq. (4) and incorporate the symmetry projection in the QMC, computing the objects with numerical integrations over the projection operators [27]. The computational cost is then proportional to that for a simple HF trial wave function, with a proportionality constant which depends on the symmetries involved but only grows modestly with system size (e.g., linearly for translation). This is a very appealing feature of the symmetry-projected HF wave functions as $\left|\Psi_{T}\right\rangle$, in contrast with, for instance, the CASSCF type of wave functions, and offers the promise of scalable calculations with our approach to reach large system sizes.

\section{CONCLUSION}

We have studied symmetry-projected HF wave functions as trial wave functions for CPMC. The symmetries are restored by projecting from a UHF- or GHF-type wave function, minimizing the variational energy after the projection. This gives a hierarchy of wave functions with increasing quality. The CPMC results using these as trial wave functions generally become increasingly more accurate, and the Monte Carlo variance becomes increasingly smaller for the same amount of samples. It is shown that CPMC largely restores size extensivity in symmetry-projected HF wave functions. At half filling in the Hubbard model, projected wave functions with spin symmetry provide an excellent description of the systems. Away from half filling, the projected wave functions with space-group symmetry and spin symmetry lead to highly accurate results, often with the constrained-path systematic error at less than $0.1 \%$ in the correlation energy. Wave functions projected from a GHF-type reference tend to perform even better compared with the ones projected from UHF types.

As discussed above, the symmetry-projected wave function can be implemented in CPMC as trial wave functions with a computational cost which scales modestly with system size. The number of Slater determinants increases linearly with system size for LM and SG projections. The size of an accurate grid used in the $S_{z}$ or full-spin (S) projection depends weakly (sublinearly) on system size. Although we have limited our study to spin-balanced systems $\left(N_{\uparrow}=N_{\downarrow}\right)$, the formalism straightforwardly applies to the more general case of finite spin polarization. The development in this paper is thus potentially a scalable and systematically improvable quantum Monte Carlo approach for extended systems. Moreover, one can further increase the quality of the symmetry-projected trial wave function by doing particle-number symmetry breaking or by incorporating multicomponent expansions such as those considered in our previous work [29]. We finally note that the current developments in CPMC have paved the way for accurate many-body calculations of Hamiltonians with spin-orbit coupling.

\section{ACKNOWLEDGMENTS}

We thank S. Chiesa, F. Ma, W. Purwanto, and E. Walter for helpful discussions. This research was supported by the US Department of Energy (Grants No. DE-SC0008627 and

TABLE II. CPMC ground-state energy of the Hubbard model in square lattices. Symmetry-projected HF trial wave functions are used; the ground state in all cases corresponds to a singlet transforming as the $B_{1}$ irrep of the $C_{4 v}$ group with momentum $(0,0)$. Results using the UHF trial wave function are listed for comparison. The last column is the release constraint [28] results (using the CASSCF trial wave functions), which are the best estimate of the exact ground-state energy. The statistical error bars in the QMC results are on the last digit and shown in parentheses.

\begin{tabular}{llllllll}
\hline \hline System & $U$ & UHF & S-UHF & LM,S-UHF & SG,S-UHF & SG, $S_{z}$-GHF & Exact \\
\hline $6 \times 6,12 \uparrow 12 \downarrow$ & 4 & $-42.621(6)$ & $-42.605(3)$ & $-42.633(3)$ & $-42.670(2)$ & $-42.679(1)$ & $-42.669(8)$ \\
$6 \times 6,12 \uparrow 12 \downarrow$ & 8 & $-36.68(7)$ & $-36.20(2)$ & $-36.94(1)$ & $-37.188(8)$ & $-37.383(4)$ & $-37.41(6)$ \\
$8 \times 8,22 \uparrow 2 \downarrow$ & 4 & $-75.886(8)$ & $-75.875(7)$ & $-75.900(6)$ & $-75.904(3)$ & $-75.893(9)$ \\
\hline \hline
\end{tabular}


No. DE-FG02-11ER16257) and the NSF (Grant No. DMR1006217). The work at Rice University was supported by the US Department of Energy, Office of Basic Energy Sciences, Heavy Element Chemistry program (Grant No. DEFG0204ER15523) and DOE-CMCSN (Grant No. DE-SC0006650). We also acknowledge an INCITE award for computing using resources of the Oak Ridge Leadership Computing Facility at the Oak Ridge National Laboratory, which is supported by the Office of Science of the US Department of Energy under Contract No. DE-AC05-00OR22725 and CPD at the College of William and Mary. G.E.S. acknowledges support as a Welch Foundation Chair (Grant No. C-0036).
[1] J. G. Bednorz and K. A. Müller, Z. Phys. B 64, 189 (1986).

[2] E. Dagotto, Science 309, 257 (2005).

[3] M. H. Y. Kamihara, T. Watanabe, and H. Hosono, J. Am. Chem. Soc. 130, 3296 (2008).

[4] G. R. Stewart, Rev. Mod. Phys. 83, 1589 (2011).

[5] W. L. McMillan, Phys. Rev. 138, A442 (1965).

[6] D. Ceperley, G. V. Chester, and M. H. Kalos, Phys. Rev. B 16, 3081 (1977).

[7] C. J. Umrigar, K. G. Wilson, and J. W. Wilkins, Phys. Rev. Lett. 60, 1719 (1988).

[8] R. J. Bartlett and M. Musiał, Rev. Mod. Phys. 79, 291 (2007).

[9] S. R. White, Phys. Rev. Lett. 69, 2863 (1992).

[10] U. Schollwöck, Rev. Mod. Phys. 77, 259 (2005).

[11] U. Schollwöck, Ann. Phys. 326, 96 (2011).

[12] R. Blankenbecler, D. J. Scalapino, and R. L. Sugar, Phys. Rev. D 24, 2278 (1981).

[13] G. Sugiyama and S. Koonin, Ann. Phys. 168, 1 (1986).

[14] S. Zhang, in the OpenAccess book, Emergent Phenomena in Correlated Matter: Modeling and Simulation Vol. 3, edited by E. Pavarini, E. Koch, and U. Schollwöck, http://www.condmat.de/events/correl13 (2013).

[15] S. Zhang, J. Carlson, and J. E. Gubernatis, Phys. Rev. B 55, 7464 (1997).

[16] C.-C. Chang and S. Zhang, Phys. Rev. B 78, 165101 (2008).

[17] S. Zhang and H. Krakauer, Phys. Rev. Lett. 90, 136401 (2003).

[18] W. Purwanto, H. Krakauer, and S. Zhang, Phys. Rev. B 80, 214116 (2009).

[19] C.-C. Chang and S. Zhang, Phys. Rev. Lett. 104, 116402 (2010).

[20] W. Purwanto, H. Krakauer, Y. Virgus, and S. Zhang, J. Chem. Phys. 135, 164105 (2011).

[21] P. Ring and P. Schuck, The Nuclear Many-Body Problem (Springer-Verlag, New York, 1980).
[22] T. Mizusaki and M. Imada, Phys. Rev. B 69, 125110 (2004).

[23] F. F. Assaad, P. Werner, P. Corboz, E. Gull, and M. Troyer, Phys. Rev. B 72, 224518 (2005).

[24] K. W. Schmid, T. Dahm, J. Margueron, and H. Müther, Phys. Rev. B 72, 085116 (2005).

[25] Q. Jie, Phys. Rev. E 77, 026705 (2008).

[26] D. Tahara and M. Imada, J. Phys. Soc. Jpn. 77, 114701 (2008).

[27] R. Rodríguez-Guzmán, K. W. Schmid, C. A. Jiménez-Hoyos, and G. E. Scuseria, Phys. Rev. B 85, 245130 (2012).

[28] H. Shi and S. Zhang, Phys. Rev. B 88, 125132 (2013).

[29] R. Rodríguez-Guzmán, C. A. Jiménez-Hoyos, R. Schutski, and G. E. Scuseria, Phys. Rev. B 87, 235129 (2013).

[30] G. E. Scuseria, C. A. Jiménez-Hoyos, T. M. Henderson, K. Samanta, and J. K. Ellis, J. Chem. Phys. 135, 124108 (2011).

[31] C. A. Jiménez-Hoyos, T. M. Henderson, T. Tsuchimochi, and G. E. Scuseria, J. Chem. Phys. 136, 164109 (2012).

[32] J. Bonnard and O. Juillet, Phys. Rev. Lett. 111, 012502 (2013).

[33] J. Hubbard, Proc. R. Soc. London Ser. A 276, 238 (1963).

[34] H. F. Trotter, Proc. Amer. Math. Soc. 10, 545 (1959).

[35] M. Suzuki, Comm. Math. Phys. 51, 183 (1976).

[36] J. E. Hirsch, Phys. Rev. B 28, 4059 (1983).

[37] D. R. Hamann and S. B. Fahy, Phys. Rev. B 41, 11352 (1990).

[38] S. R. White, D. J. Scalapino, R. L. Sugar, E. Y. Loh, J. E. Gubernatis, and R. T. Scalettar, Phys. Rev. B 40, 506 (1989).

[39] M. Calandra Buonaura and S. Sorella, Phys. Rev. B 57, 11446 (1998).

[40] D. A. Huse, Phys. Rev. B 37, 2380 (1988).

[41] E. Y. Loh, J. E. Gubernatis, R. T. Scalettar, S. R. White, D. J. Scalapino, and R. L. Sugar, Phys. Rev. B 41, 9301 (1990).

[42] J. Carlson, J. E. Gubernatis, G. Ortiz, and S. Zhang, Phys. Rev. B 59, 12788 (1999).

[43] W. Purwanto and S. Zhang, Phys. Rev. E 70, 056702 (2004). 